\title{
Sizing and Lifecycle Cost Analysis of an Ares V Composite Interstage
}

\author{
Troy Mann ${ }^{\dagger}$, Stan Smeltzer ${ }^{\star}$, Ray Grenoble ${ }^{\S}$, Brian Mason ${ }^{* *}$ \\ NASA Langley Research Center, Hampton, VA, 23681 \\ (757) 864-9515 \\ Sev Rosario ${ }^{\dagger \dagger}$ \\ Analytical Mechanics Associates Inc. (AMA), Hampton, VA, 23681 \\ Bob Fairbairn ${ }^{*+}$ \\ Vigyan, Hampton, VA, 23681
}

\begin{abstract}
The Interstage Element of the Ares V launch vehicle was sized using a commercially available structural sizing software tool. Two different concepts were considered, a metallic design and a composite design. Both concepts were sized using similar levels of analysis fidelity and included the influence of design details on each concept. Additionally, the impact of the different manufacturing techniques and failure mechanisms for composite and metallic construction were considered. Significant details were included in analysis models of each concept, including penetrations for human access, joint connections, as well as secondary loading effects. The designs and results of the analysis were used to determine lifecycle cost estimates for the two Interstage designs. Lifecycle cost estimates were based on industry provided cost data for similar launch vehicle components. The results indicated that significant mass as well as cost savings are attainable for the chosen composite concept as compared with a metallic option.
\end{abstract}

\section{Nomenclature}

$\mathrm{Al}-\mathrm{Li}=$ Aluminum-Lithium Alloy 2195

DDT\&E = Design, Development, Test, and Evaluation

EDS $=$ Earth Departure Stage

HLLV = Heavy Lift Launch Vehicle

IMLEO = Injected Mass to LEO

$\mathrm{KSC} \quad=$ Kennedy Space Center

LaRC = Langley Research Center

LEO $=$ Low Earth Orbit

MSFC $=$ Marshall Space Flight Center

NASA $=$ National Aeronautics and Space Administration

$\mathrm{OHC}=$ Open Hole Compression

RCS $=$ Reaction Control System

\footnotetext{
† Senior Structural Analyst, Structural and Thermal Systems Branch, MS/431, AIAA Senior Member.

* Deputy Manager, Ares Project Office, MS/208, AIAA Associate Fellow.

${ }^{\S}$ Senior Structural Analyst, Durability, Damage Tolerance, and Reliability Branch, MS/188E.

** Senior Structural Analyst, Durability, Damage Tolerance, and Reliability Branch, MS/188E, AIAA Senior Member.

${ }^{\dagger}$ Senior Project Engineer (AMA, Inc.), Structural and Thermal Systems Branch, MS/431.

\$ Senior Cost Analyst, Systems Management Office, MS/020
} 


\section{Introduction}

$\mathrm{T}$ HE National Aeronautics and Space Administration (NASA) is currently investigating long duration manned missions outside low earth orbit (LEO). These missions are anticipated to require significantly larger launch vehicles than are currently available (commercially or otherwise). Significant development efforts have taken place to date, and include sizing components from representative launch vehicles necessary for these missions. While these efforts have demonstrated the potential for composite structures to decrease the mass of such a launch vehicle, it is important to consider the costs associated with the two primary material selection options: metallic and composite. In order to compare the manufacturing and fabrication techniques appropriately for these two material options, a segment of a representative launch vehicle was evaluated using composite materials and metallic materials. The costs associated with manufacturing technology development, production, insight/oversight, and unrealized mass benefits were then calculated and compared. The present paper sets the stage for a lifecycle cost comparison between metallic and composite manufacturing and fabrication methods by evaluating two competing Interstage designs, with a further objective to determine the manufacturing and fabrication method that results in the design with the least mass. These baseline representations are then used to calculate lifecycle costs. In this paper, the composite and metallic design concepts are compared using mass, technology and manufacturing development costs, and recurring costs.

\section{Ares V Background}

The Ares V launch vehicle was originally conceived as the heavy-lift component of the Ares launch vehicles for missions to the moon as well as alternate destinations. Ares $\mathrm{V}$ was designed to be approximately 360 feet tall and weigh 7.4 million pounds at launch while delivering more than 220,000 pounds of cargo to $\mathrm{LEO}^{1}$. The Ares V, shown in Fig. 1, consists of a shuttle-derived first stage with two attached solid rocket motors. The upper stages (the Earth Departure Stage (EDS) and the Shroud) are connected to the first stage through the Interstage element, sized and evaluated in this study.

While several design reference missions were considered through the life of the Ares V Project, the EDS generally was expected to separate from the vehicle at an altitude of approximately 450,000 feet at a speed of Mach $8.8^{1}$. As a result, the Interstage would be required to transfer loads from the payload Shroud and payload to the first stage structures during launch and ascent.

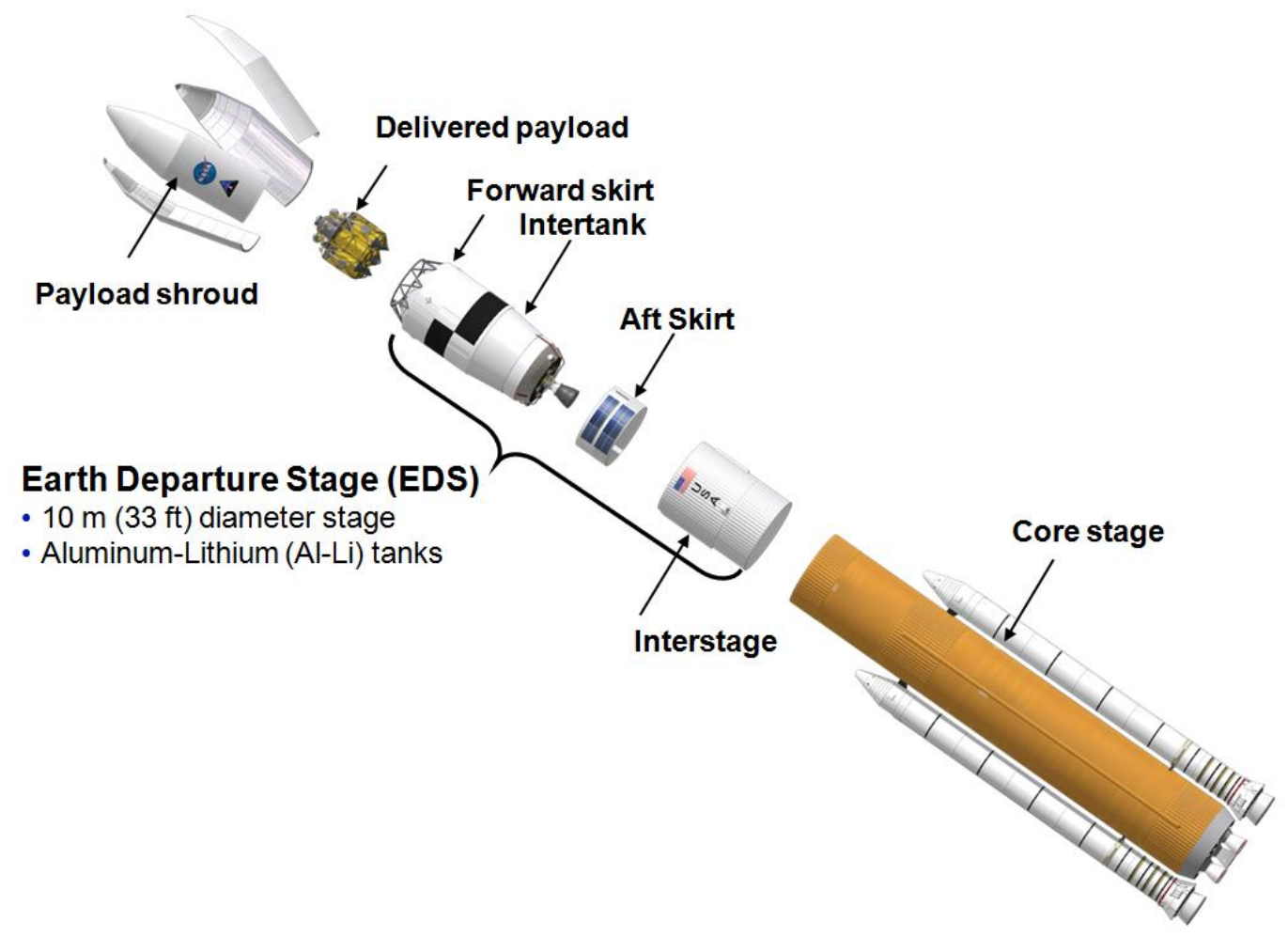

Figure 1. Ares V Conceptual Design. 
The Constellation Program that included Ares V was canceled in October of 2010. However, the future vision of NASA includes destinations for manned and unmanned exploration that will likely require launch vehicles of similar magnitude to Ares V. The analysis included in this paper will still be relevant for dry structures subject to similar loading conditions and environments. When comparing metallic and composite concepts the trends have shown consistency through multiple design cycles. These trends are anticipated to remain similarly consistent when considering different launch vehicles of this magnitude.

\section{Analysis Ground Rules and Assumptions}

Two Interstage designs were evaluated in this study: a metallic (aluminum-lithium 2195 alloy [Al-Li]) hat stiffened design and a composite sandwich design that utilized IM7/8552 carbon-epoxy facesheets and a Hexcel aluminum honeycomb core. The analysis was intended to provide minimum mass results for both concepts despite the different analysis and manufacturing and fabrication methods. Where possible, the requirements and assumptions were held constant between the two designs for a fair comparison of the results.

\section{A. Buckling and Crippling}

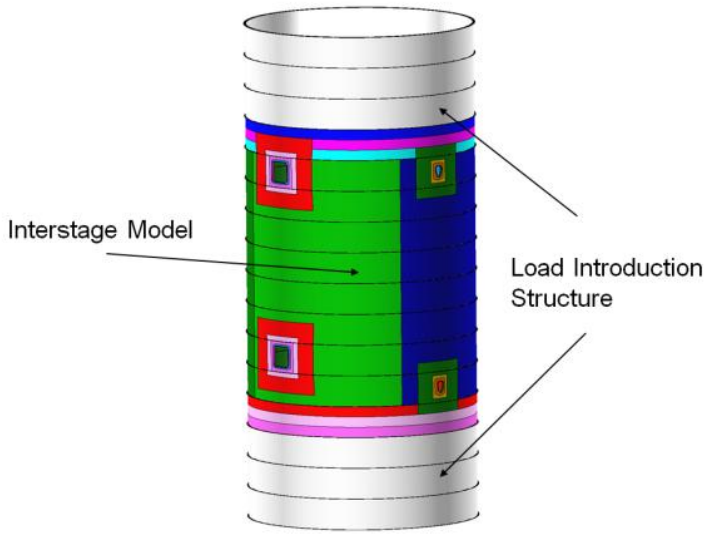

Figure 2. Interstage Finite Element Model

Shell buckling knockdown factors for the two designs were consistent with Ref. 3. Crippling and cripplingbuckling interaction were considered failure mechanisms only for the metallic structures because the material can yield; this wasn't an option for the composite structures considered herein.

\section{B. Material Geometric Constraints}

The minimum gage for the Al-Li material was 0.06 in. while the composite minimum gage was limited to 8 plies with a minimum of $10 \%$ of the plies in any one of the four principal directions $(0,+45,-45,90)$.

\section{Standardization of Finite Element Boundary Conditions}

Modeling only the structure of interest generally results in high loads being present in the vicinity of the model boundaries. Subsequently, sizing analyses using these loads will yield overly conservative structures. To avoid being overly conservative, additional element groups at the ends of the structure were included to represent the adjacent structure. Attachment rings between major components were also included in the analysis to provide a representative stiffness. An example of the representative load introduction structures and boundary conditions used in the analysis is shown in Fig. 2.

\section{Material Properties}

Al-Li material properties were provided in Ref. 4. IM7/8552 material values were provided by the Marshall Space Flight Center (MSFC) (Ref. 5), which included representative open-hole compression (OHC) values for this material system. The use of $\mathrm{OHC}$ values in the analysis for the composites certainly yielded conservative results, but do allow for a more relaxed damage control plan than would be required for pristine material values.

\section{E. Factors of Safety}

Reference 6 was used to determine factors of safety applied to the different concept. Specifically, ultimate and limit factors of safety of 1.4 and 1.25, respectively were applied to the metallic concept while 1.4 and 1.0 for ultimate and limit factors of safety, respectively were applied to the composite concept. Additionally, a factor of 2.0 was applied to discontinuities in the composite concept per Ref. 5.

\section{F. Loads}

Loads applied to the Interstage were provided by the Ares V Project Office as section component loads (axial force, shear force, bending moment) at each section along the length of the vehicle, for multiple load cases. The maximum values for these forces along the length of the Interstage were applied to a rigid element attached to the top of the load introduction structure at the top of the model. The translational degrees of freedom at the bottom of 
the model were constrained axially. The lateral loads were applied to the model in multiple load cases at different angles with respect to the central axis because of the asymmetry in the model due to the cutouts.

\section{Initial Sizing}

Hypersizer $\AA^{\S \S}$ software was used to size the panels and beams that make up the Interstage. The software allows the user to specify a wall concept type, materials, and possible geometric parameters for panels and beams. Multiple failure calculations are then made given the loads applied to the components that make up a model. Starting with the lightest permutation multiple failure criteria are checked. If the component 'fails' these checks with negative margins of safety, the next permutation is evaluated until the lightest permutation that has positive margins of safety for all of the possible failure modes is found. This process can then be iterated to determine any impact of load redistribution on the model.

The metallic version of the Interstage generally benefits from the use of ring frames along the length to provide buckling stability. A study was initially conducted to determine the optimum ring-frame spacing for the large acreage portions of the Interstage barrel. A finite element representation of the Interstage was created and subjected to axial and lateral launch and ascent conditions. Ring frames were then included at different spacing along the length of the Interstage. The ring frames were sized using Shanley's criteria ${ }^{2}$ to ensure a global buckling pattern with node lines at the ring frame locations. The panels between the ring frames were then sized appropriately for the applied loads and local buckling constraints. The total mass for the Interstage was then calculated.

The results of the ring frame spacing analysis are shown in Fig. 3. With no ring frames, the acreage skin mass is relatively high as the buckling length for the Interstage is very long. By introducing ring frames and decreasing the ring frame spacing, the areal mass of the acreage skin decreases. However, as more and more frames are introduced, the mass contribution from the ring frames increases to a point where the benefit of the increased stiffness from the ring frames is overcome by the ring frame mass impact. As a result, there is an optimum frame spacing that yields minimum mass for this concept. The sensitivity to ring frame spacing at the optimum frame spacing is relatively small since there is a relatively flat line in the total mass values as shown in Fig. 3. The maximum frame spacing in this area of optimum mass was chosen knowing that the manufacturing cost will be less. Analysis of the composite version of the Interstage showed that the addition of ring frames did not provide mass savings (ring frames were included at the ends, with more detail provided in the detailed analysis).

\section{Detailed Sizing Analysis}

While the initial sizing indicated a significant mass savings afforded by using composite materials $(>40 \%)$, it was anticipated that some of these savings would be compromised when more detail was included in the model. In particular, the higher factor of safety required for discontinuities was expected to increase the mass contribution at locations for penetrations required for ingress/egress and venting. Additionally, the inclusion of metallic joints at the upper and lower interfaces on the composite Interstage will require build-ups that will increase the mass locally. Rather than relying on personal engineering judgment in these areas, an attempt was made to include all of these design details in the analysis for the two Interstage designs.

\section{A. Cutouts}

Human access to the components interior to the Interstage shell (during fabrication, integration, and potentially on the launch pad) necessitated the inclusion of two doors located at either end of the Interstage barrel. The doors were sized following guidelines provided by operations personnel at the Kennedy Space Center (KSC). Additionally, two vent/purge lines were included in the model, also based on

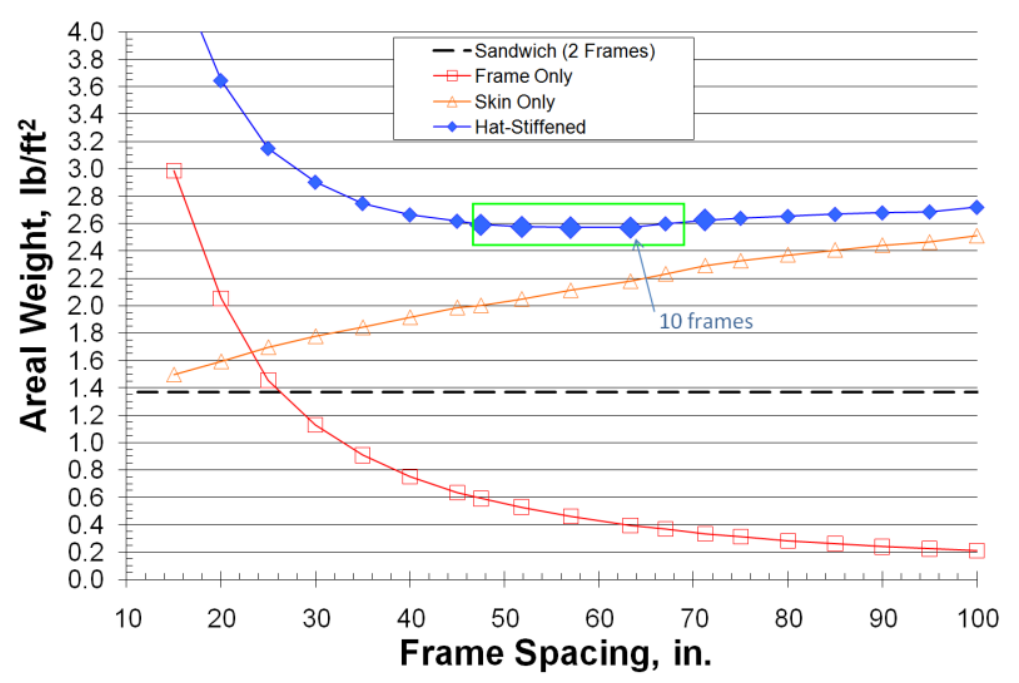

Figure 3. Optimum Ring Frame Spacing Ares V Interstage Metallic Concept

$\S \S$ This is not an endorsement by the National Aeronautics and Space Administration (NASA)

4

American Institute of Aeronautics and Astronautics 
the feedback from KSC personnel. Those sections of the model immediately surrounding the penetrations were separated to allow appropriate sizing of the buildup area required for load redistribution while the penetrations were assumed to carry no load (a conservative assumption). The model with the access doors and vent/purge holes is shown in Fig. 2. The resulting model was no longer axisymmetric with the inclusion of the penetrations. Since the loads provided for sizing did not assume a vehicle attitude, multiple load cases were now needed to ensure maximum loading throughout the model. The lateral shear force and bending moment were rotated around the central axis of the Interstage to force loading directly over the penetrations.

Sizing the model with the modifications for cutouts yielded acreage panels that weighed $1.1 \mathrm{lb} / \mathrm{ft}^{2}$ and $2.0 \mathrm{lb} / \mathrm{ft}^{2}$ for the composite and metallic concepts, respectively. This yields total panel masses of $7722 \mathrm{lbm}$ and 14,420 lbm for the composite and metallic concepts, respectively. The beam masses for the metallic concept (3093 lbm) were significantly larger than the beam masses for the composite concept $(336 \mathrm{lbm})$ because of the additional ring frames necessary for buckling stability (in the metallic design). The total masses for the two concepts were $8232 \mathrm{lbm}$ for the composite and 17,600 lbm for the metallic concept.

Comparing the sizing results with the initial analysis results afforded some insight into the impact of the cutouts. The portal openings added approximately $87 \mathrm{lbm}$ (each) for the composite concept, but only $45 \mathrm{lbm}$ for the metallic design. The vent/purge cutouts added only $16.8 \mathrm{lbm}$ and $13.5 \mathrm{lbm}$ for the composite and metallic concepts, respectively.

\section{B. Joint Details}

The Interstage attaches at the forward end to the EDS Intertank and the aft end of the Interstage attaches to the core stage section (see Fig. 1) at the forward end of the Instrument Unit. Initial analysis of the Interstage did not consider these attachments. However, the attachments and any additional buildup at these locations must be accounted for in any mass comparisons. A general assumption was made that a flange type connection would be used for both the metallic and composite concepts. However, implementation of this flange required much different design details for the two concept types.

The metallic joint design is relatively simple since the flange can be welded directly to the acreage material using a friction stir weld. The weld tool requires a two inch area of material on either side of the weld without stiffeners for this operation. Mass estimates for the metallic Interstage concept were calculated assuming the crosssection concept detail shown in Fig. 4. The stiffeners were assumed to taper to a flat area two inches long. This ring is then welded to the joint ring with another two inch flat section. The flange then connects to the adjacent ring section with bolts, represented in Fig. 4 by dashed lines. A detailed finite element model of this joint attachment ring is shown in Fig. 5 and is used to size thicknesses of the flange, flats, and webs appropriately (including a knockdown factor on the material allowable properties of the weld material).

The composite joint had additional complications when compared with the metallic joint. The composite acreage panels were assumed to be closed out at the ends with higher density core material. Additional plies were added in the area of the high density core to react bearing loads from the fasteners that attach the pocket of the joint to the composite panel. The number of plies required was calculated by selecting a bolt diameter and calculating the bearing load on the facesheets (given the applied shear load). By selecting a number of different bolt sizes, the sensitivity of the joint mass was evaluated. The additional detail in the panel that was evaluated in the composite joint area is shown in Fig. 6.

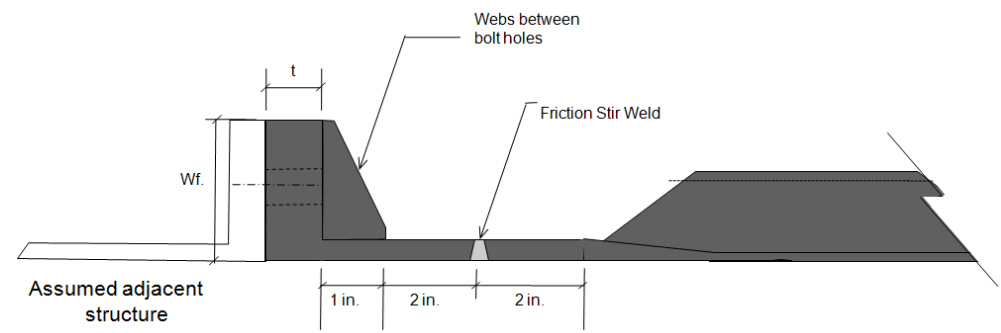

Figure 4. Metallic End-Ring Cross-Sectional Geometry Assumptions

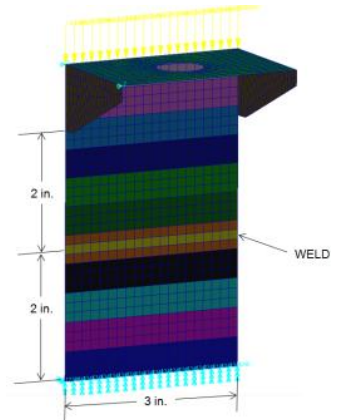

Figure 5. Detailed Metallic Joint Model 


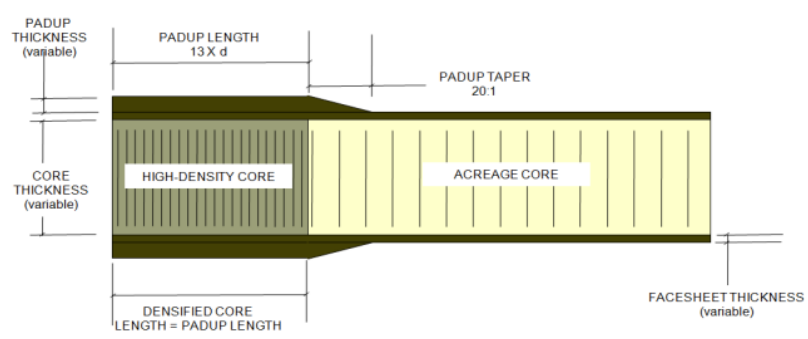

Figure 6. Detailed Composite Panel Buildup at Joints

The end ring for the composite panels is shown in Fig. 7 and consists of a flange, similar to the metallic joint concept, with a pocket that fits over the modified panel. The geometry of the end ring was calculated given the bolt diameter and applied loads using a strength of materials approach.

Five different bolt sizes were evaluated ranging in size from 0.25 in. to 0.5 in. The results of the joint mass details are listed in Table 1. The smallest bolt diameter yielded the minimum mass, but also required a large number of fasteners. It was assumed that the large number of bolts would increase costs, so a compromise solution was also evaluated. By increasing the pad-up thickness, fewer bolts could be used (without reaching the bearing strength of the facesheet plies) with an inherent mass penalty ( 20\% increased mass for the composite joint design).

Table 1: Interstage Joint Mass Details

\begin{tabular}{|c|c|c|c|c|c|c|c|c|}
\hline $\begin{array}{c}\text { Structural } \\
\text { Interface }\end{array}$ & Design & $\begin{array}{c}\text { Pad-up } \\
\text { Thickness } \\
\text { (in) }\end{array}$ & $\begin{array}{c}\text { Number } \\
\text { of } \\
\text { Fasteners }\end{array}$ & $\begin{array}{c}\text { Pad-up } \\
\text { Mass } \\
(\mathbf{l b m})\end{array}$ & $\begin{array}{c}\text { Core } \\
\text { Densification } \\
(\mathbf{l b m})\end{array}$ & $\begin{array}{c}\text { Fastener } \\
\text { Mass } \\
(\mathbf{l b m})\end{array}$ & $\begin{array}{c}\text { Ring Mass } \\
\text { (lbm) }\end{array}$ & $\begin{array}{c}\text { Total Mass } \\
\text { (lbm) }\end{array}$ \\
\hline \multirow{2}{*}{$\begin{array}{c}\text { Composite } \\
\text { Forward } \\
\text { Flange }\end{array}$} & $\begin{array}{c}\text { Minimum } \\
\text { Mass }\end{array}$ & 0.01 & 2294 & 4.8 & 73.7 & 76.6 & 413.9 & 569.9 \\
\cline { 2 - 9 } & Compromise & 0.08 & 1162 & 46 & 73.7 & 40.1 & 455.3 & 615.1 \\
\hline $\begin{array}{c}\text { Composite } \\
\text { Aft Flange }\end{array}$ & $\begin{array}{c}\text { Minimum } \\
\text { Mass }\end{array}$ & 0.01 & 3237 & 4.8 & 73.7 & 108.1 & 457 & 643.6 \\
\hline $\begin{array}{c}\text { Metallic } \\
\text { Forward } \\
\text { or Aft }\end{array}$ & & 0.09 & 1374 & 53 & 73.7 & 47.6 & 507.5 & 681.8 \\
\hline
\end{tabular}

\section{Secondary Loading Effects}

Previous experience has shown the need to consider any significant point loads, attachments, or secondary loads, particularly for composite concepts. In order to capture any impact due to secondary thrust loads (ullage settling motors, reaction control system (RCS) firings, etc.) a detail model, shown in Fig. 8, was developed that allowed for the sizing of a cutout (and surrounding structure) due to these loads. Thrust loads for ullage settling and RCS firing were difficult to determine given the early stage of design, so the thrust loads were assumed to be three times larger than those used for the Saturn S-IVB upper stage. The panel section was then sized with and without the thrust loads to determine the impact of including this detail. For the thrust level applied and the loads that sized the acreage panels, no mass increase was required to react the secondary loads.

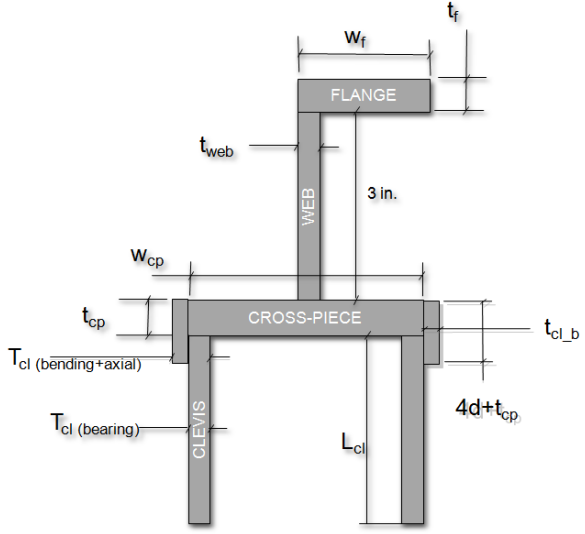

Figure 7. End-Ring Cross-Sectional Geometry for Composite Panels 


\section{Cost Analysis}

The results of the detailed sizing analysis provided a baseline design to evaluate the costs associated with the anticipated lifecycle costs for these two manufacturing and fabrication methods, from development through production. NASA Langley Research Center (LaRC) has been developing parametric cost modeling tools since 1992. These tools maximize the use of available definition information, leveraging historical design, analysis, and cost results. The tools utilize descriptive, in-depth parameter sets at a common element level. Additionally, the element level is selected at an appropriate point in the development or manufacturing process to faithfully reproduce the processes involved and remove analyst judgment as much as possible. The results provide useful feedback regarding sensitivity trends to allow for 'sanity check' evaluation of results throughout the process.

For evaluation of the Interstage geometry, the costs of individual parts, from development through fabrication, and assembly, were determined considering mechanical parameters, electrical parameters, and project unique parameters. These costs were included in assembly level fabrication, final integration in system level operations, testing, and checkout operations. Examples of the mechanical, electrical, and project unique parameters are shown in Table 2.

Table 2: Mechanical, Electrical, and Project Unique Parameters

\begin{tabular}{|l|l|l|}
\hline Mechanical Parameters & Electrical Parameters & Project Unique Parameters \\
\hline Quantity per assembly & Quantity per assembly & Engineering level \\
Mass & Component area & Engineering difficulty \\
Number parts & Component or package pins & New drawing fraction \\
Unique fraction & Component active elements & Design, Fabrication multiplier (include or not) \\
Average number instructions/part & Unique fraction & I\&T Plans fraction \\
Precision & Component type & I\&T multiplier (include or not) \\
Machinability index (Batelle scale) & Package type & Number prototype units, per phase \\
Assembly difficulty & Unit learning curve for fabrication & Specification (platform) level, per phase \\
Fraction hogout & Component boundary & Test level, per phase \\
Fraction yield loss & Integration difficulty & Multiplier, phase duration, per phase \\
Surface finish & Board type & Fabrication unit fraction of overlap within phase \\
Surface area & Board layers & Multiplier, resources, per phase \\
Optics design complexity & Total board mass & Multiplier, resources, per function \\
Unit learning curve for fabrication & Volume & Unit learning curve for fabrication, per phase \\
\cline { 2 - 2 } Integration difficulty & & Unit learning curve for engineering, per phase \\
Volume & & Schedule constraints \\
\cline { 2 - 3 } & & Multiplier on staff level penalty \\
\cline { 2 - 3 } & & Hours per labor month \\
\hline
\end{tabular}

While NASA has conducted significant technology development to enable the production of a Heavy Lift Launch Vehicle (HLLV), the manufacturing development and production will likely take place at a prime contractor location. As a result, it is necessary to have some insight into the industry manufacturing development and production costs. Upfront design decisions will drive lifecycle costs, and the manufacturing development and production costs are needed to accurately model this impact. NASA contracted with the Lockheed-Martin Corporation (Lockheed-Martin) and the Boeing Company (Boeing) to provide detailed cost models of generic Interstage designs focused specifically on composite fabrication methods. Additionally, Boeing provided cost analysis information for the Ares I Interstage design (both metallic and composite concepts), another large cylindrical barrel design, as part of the Ares I Project.

The cost analysis details provided by Lockheed-Martin and Boeing were used to verify and adjust the cost modeling software used at LaRC. The results of the industry studies were duplicated using the LaRC cost parameter tools considering similar prototype definitions, development and production schedules, technology development, material characterization, manufacturing test demonstrations, system test demonstrations, and production costs. No significant method modifications were required to reach similar cost estimates compared with the industry results. 


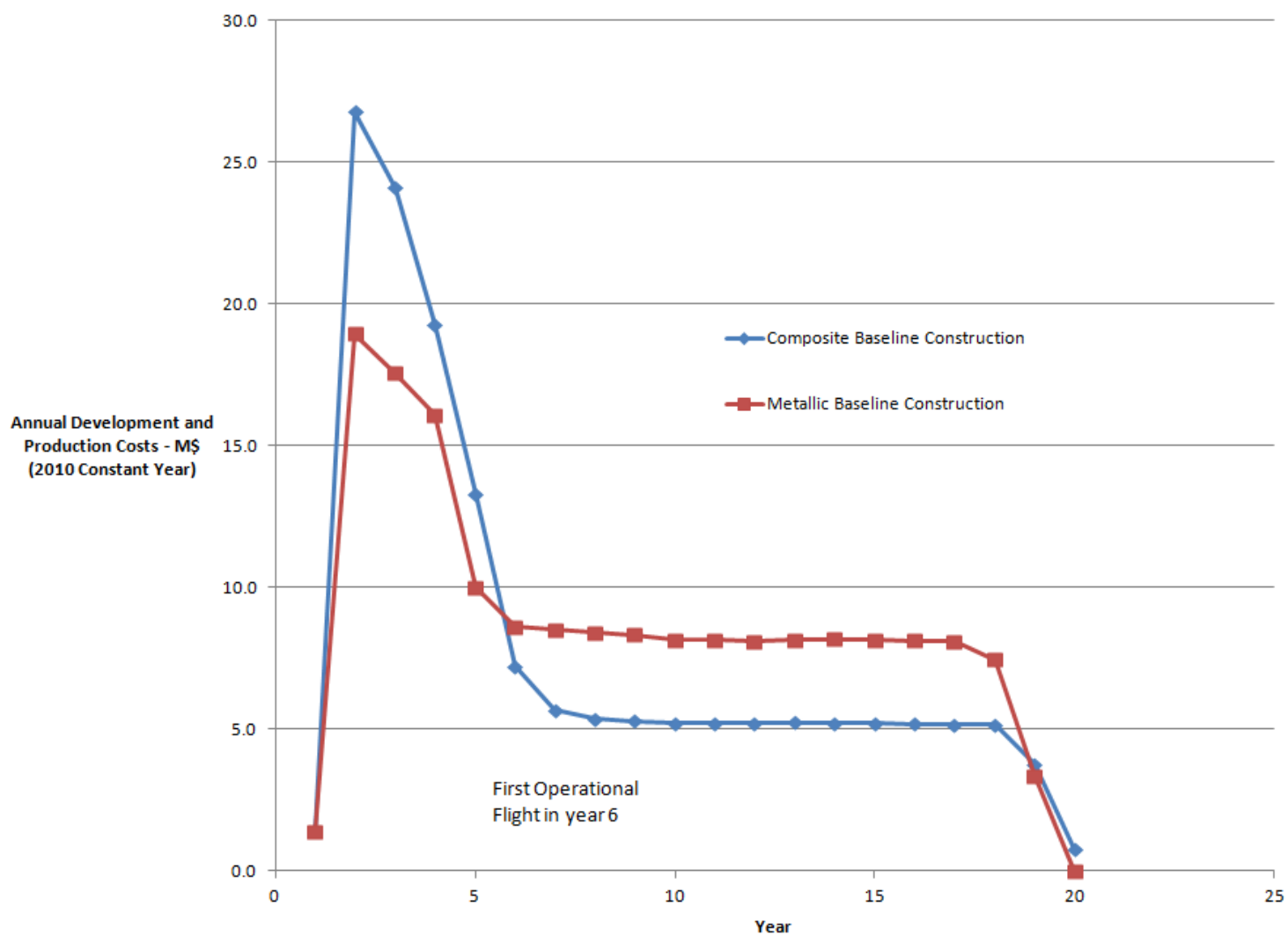

Figure 9: Annual Development and Production Costs

In an attempt to further validate the tool, a meeting was held with representatives from the Marshall Space Flight Center (MSFC) who use the NASA/Air Force Cost Model (NAFCOM) database. The results determined using the LaRC parameter cost tools showed similar trends to those evaluated previously with NAFCOM. Additionally, the extra scrutiny provided confidence that there were no significant costs left out of the evaluation or improperly estimated.

A cost model for the Ares V Interstage was developed using the previously described tools. The design details provided in Sections IV and V drove the cost model development. The cost model provided production costs, staff levels, and design, development, test, and evaluation (DDT\&E) costs. The annual development and production costs are shown in Fig. 9. The cost model results in Fig 9 indicate that initial costs were significantly higher for the development of the composite concept. However, the production costs for the composite concept ( $\$ 5 \mathrm{M} / \mathrm{unit})$ were dramatically lower than the manufacturing and fabrication costs for the metallic concept ( $\$ 8 \mathrm{M} / \mathrm{unit})$. Boeing and Lockheed-Martin found similar trends in their evaluation of their composite and metallic concepts. This trend was generally governed by lower part counts and decreased touch labor for the composite concept compared with the metallic concept. This trend is an important consideration when determining the preferred manufacturing and fabrication techniques.

The results from the cost analysis provided the information necessary to determine lifecycle costs for the Interstage, from a system perspective. However, the mass differences seen between the metallic and composite concepts did not allow a consistent comparison between the two concepts. At a system level, the capability of the launch vehicle would be impacted by the decision to use one or the other manufacturing and fabrication techniques because of the change in inserted mass to low-Earth orbit (IMLEO). Flying a less capable vehicle will require additional flights in order to complete an assigned mission successfully. An attempt was made to determine the impact of this effect at the system level by converting the difference in IMLEO to lifecycle costs. First, changes in mass at the Interstage level do not correlate one-to-one with changes in IMLEO because the Interstage separates from the launch vehicle prior to orbit insertion. To account for this, a gear ratio of 0.2 was utilized following trajectory analysis at the system level. In essence, this implies that a one-pound change in the mass of the Interstage will result in a 0.2-pound change in the IMLEO. Two flights per year were estimated as the flight manifest. 
Multiplying the Interstage mass difference, the gear ratio, and the flight rate together provides the difference in IMLEO per annum between a vehicle that uses a metallic Interstage and one that uses a composite Interstage.

The remaining piece of information required to determine the cost impact of the IMLEO change is the cost to raise the mass difference to orbit, $\alpha_{\mathrm{LEO}}$. Such a cost is difficult to come by and is often used as an estimate of the capability of launch systems. The Futron Corporation attempted to evaluate this metric in 2002 for a number of different launch vehicles and orbits ${ }^{7}$. The estimates for this value vary widely with different organization and different launch vehicle sizes. The best estimate for the cost to launch a pound of payload to orbit from this study that would correlate with a HLLV is approximately $\$ 5000 / \mathrm{lbm}$ (inflation adjusted to year 2010 dollars). Multiplying the IMLEO difference per year with this cost to orbit provides a system level estimate of the cost impact of the different Interstage concepts. This cost is called the unrealized mass benefit (UMB).

Adding the development and production costs over the life of the system with the UMB is shown in Fig. 10, significant cost savings can be realized with the composite version of the Interstage as conceived here. Using the nominal costs for $\alpha_{\mathrm{LEO}}$, approximately $\$ 172$ million dollars are saved with the composite concept over the life of the system. While the results are relatively sensitive to $\alpha_{\mathrm{LEO}}$, the total cost savings are significant even with dramatic (and optimistic) changes to this value. For a factor of 5 improvement in $\alpha_{\text {LEO }}$ (to $\$ 1000 /$ pound) over the state of the art, the composite concept still saves over $\$ 45$ million dollars over the life of the system in the results shown in Fig. 10.. The slope change at the end plots in Fig. 10 is because there is only one unit manufactured in the final year.

The lifecycle cost results show that the UMB is important when considering the system impact of mass changes in the design. The design and analysis of the Interstage includes significant detail regarding design details that tend to increase mass in a composite design. However, many people still approach composites with trepidation, particularly when considering mass estimates at an early stage in the design. To evaluate the impact of differences in the design results, an additional composite design was considered that showed significantly smaller mass benefits compared with the metallic design. These results are shown in Fig. 11. While the baseline composite design weighed approximately $35 \%$ less than the metallic design, the second composite design was assumed to be a more modest 20\% lighter than the metallic design. The impact of this additional mass is seen in the UMB but does not alter the production cost per unit. The baseline composite design yields approximately $\$ 172$ million over the life of

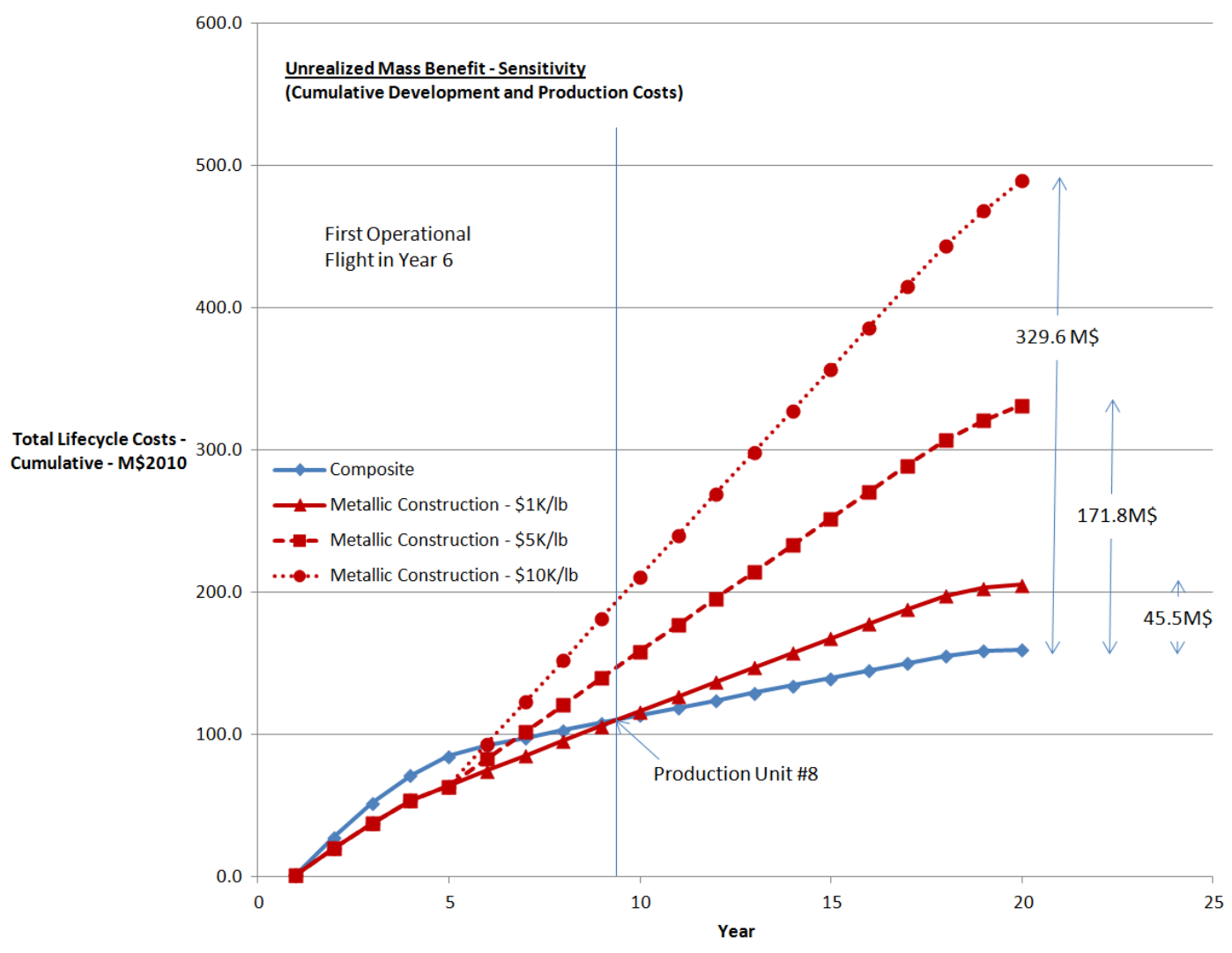

Figure 10: Total Lifecycle Costs - UMB Sensitivity 
the system while the less capable composite design still yields over $\$ 104$ million dollars in savings over the life of the system as shown in Fig. 11.

Clearly, significant costs can be saved by utilizing composite structures as detailed here. The Interstage structure analyzed is generally lightly loaded. As a result, the capabilities of a composite concept are more suited to this structure and yield lower masses. The cost analysis shows that the initial costs associated with the composite fabrication are significantly larger than those associated with the metallic concept. However, the production costs for the composite concept are significantly lower compared with the metallic concept. The implication is that for design solutions that only require a few units, it might not be cost effective to invest in the initial capital costs associated with composite concepts. However, if several units are required for the system, the initial investments will be quickly recouped by savings in fabrication costs. By considering the system level costs associated with the reduced capability of a system (UMB) that uses a heavier (metallic) Interstage, the differences are amplified.

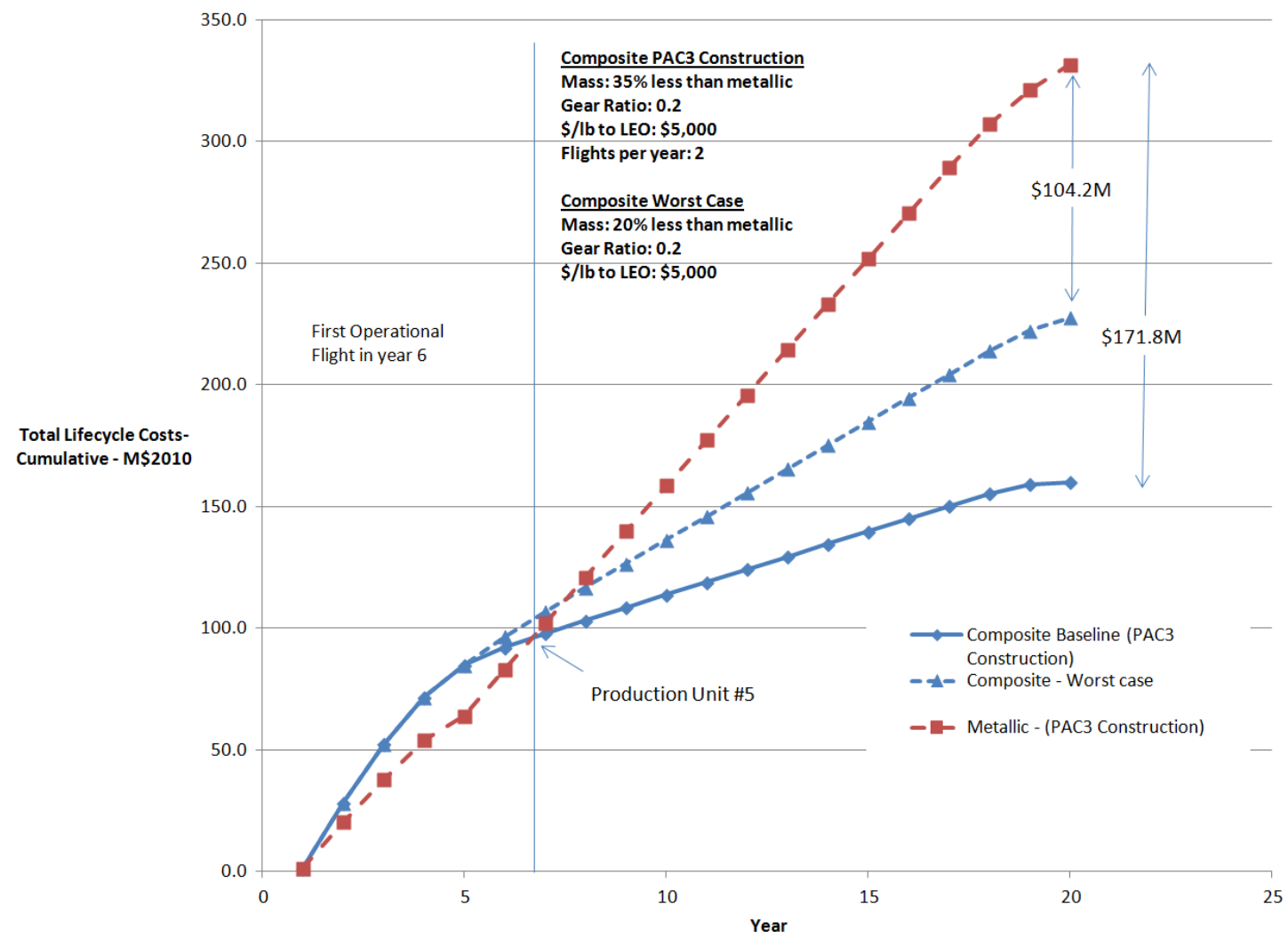

Figure 11: Total Lifecycle Costs - Composite Mass Sensitivity 


\section{Summary}

Detailed design and analysis results revealed significant mass savings ( $\sim 35 \%)$ for a composite Ares V Interstage concept as compared to a similarly designed metallic Interstage concept. The detailed analysis of the initial development costs shows significant upfront costs for a composite concept that are not required for a metallic concept. However, annual production costs for the composite concept are approximately $45 \%$ lower than the production costs of the metallic concept. These production cost differences, coupled with the mass savings seen with a composite concept, correspond to significant cost savings over the life of the Interstage $(>\$ 100 \mathrm{M}$ over the life of the project), even given the additional technology development costs during the early stages of the project.

\section{References}

${ }^{1}$ Sumrall, P., and Creech, S., "Refinements in the Design of the Ares V Cargo Launch Vehicle for NASA's Exploration Strategy," AIAA /ASME/SAE/ASEE Joint Propulsion Conference and Exhibit, Hartford, Conn., 2009

${ }^{2}$ Shanley, F.R., "Simplified Analysis of General Instability of Stiffened Shells in Pure Bending," Journal of the Aeronautical Sciences, October, 1949, pp. 590-592

${ }^{3}$ Buckling of Thin-Walled Circular Cylinders, SP-8007, NASA, 1965.

${ }^{4}$ Design Values Handbook for Aluminum-Lithium 2195 Plates, Extrusions, Forgings, \& Welds, MSFC-HDBK-3513, Rev. A

${ }^{5}$ EM40 8552/IM7 Property Memo 3/14/2007

${ }^{6}$ Structural Design and Test Factors of Safety for Spaceflight Hardware, NASA-STD-5001A, 2008

${ }^{7}$ Space Transportation Costs: Trends in Price Per Pound to Orbit 1990-2000, Futron Corporation, September 6, 2002

(http://www.futron.com/upload/wysiwyg/Resources/Whitepapers/Space_Transportation_Costs_Trends_0902.pdf) 